\title{
Sindrome de burnout em profissionais da saúde: revisão integrativa
}

\author{
Layla Thamm Jarruche ${ }^{1}$, Samantha Mucci ${ }^{1}$ \\ 1. Universidade Federal de São Paulo, São Paulo/SP, Brasil.
}

\begin{abstract}
Resumo
A incidência de síndrome de burnout ou síndrome do esgotamento profissional é significativa entre trabalhadores da saúde, com impacto negativo no âmbito pessoal, institucional, governamental e no cuidado com os pacientes. Com o objetivo de investigar o campo de pesquisas brasileiras sobre o tema, foi realizada revisão integrativa da literatura selecionando 35 artigos publicados entre 2014 e 2019. Identificou-se alto índice de síndrome de burnout em profissionais da saúde, assim como alto risco de desenvolver essa síndrome e incidência de outros transtornos mentais. A maior parte das pesquisas envolve profissionais de medicina e enfermagem, apresenta a maioria dos profissionais de saúde como do sexo feminino e é desenvolvida em hospitais e unidades básicas de saúde. Ressalta-se a necessidade de desenvolver mais pesquisas na área, principalmente envolvendo outras categorias profissionais e abrangendo outros ambientes de trabalho, analisando ainda o impacto da predominância de profissionais mulheres na saúde.
\end{abstract}

Palavras-chave: Esgotamento profissional. Pessoal de saúde. Saúde do trabalhador.

\section{Resumen}

\section{Síndrome de burnout en profesionales sanitarios: revisión integradora}

La incidencia del síndrome de burnout, o síndrome de desgaste profesional, es significativa entre los trabajadores de la salud, con un impacto negativo en el ámbito personal, institucional, gubernamental y en la atención al paciente. Para investigar los estudios brasileños sobre el tema, se realizó una revisión integradora de la literatura, con 35 artículos publicados entre 2014 y 2019. Se pudo identificar una alta tasa de síndrome de burnout en los profesionales de la salud, así como un alto riesgo de desarrollar este síndrome y la presencia de otros trastornos mentales. La mayor parte de las investigaciones involucran a profesionales médicos y de enfermería, presentan la mayoría de los profesionales de la salud como mujeres y se llevan a cabo en hospitales y unidades básicas de salud. Se destaca la necesidad de más investigaciones en el área, con otras categorías profesionales y otros entornos laborales, analizando también el impacto del predominio de las mujeres entre los profesionales en salud.

Palabras clave: Agotamiento profesional. Personal de salud. Salud laboral.

\section{Abstract \\ Burnout syndrome in healthcare professionals: an integrative review}

The incidence of burnout syndrome or professional exhaustion is significant among healthcare professionals, with negative impact on the personal, institutional, governmental, and patient care spheres. Aiming to investigate Brazilian research on the topic, we conducted an integrative literature review and selected 35 articles published from 2014 to 2019. We identified a high rate of burnout syndrome in healthcare professionals, a high risk of developing this condition and other mental disorders. Most studies were conducted in hospitals and public health centers, involved health professionals and had a greater number of female professionals. We emphasize the need to conduct further studies in the field, especially regarding other professional categories and other work environments, also analyzing the impact of the predominance of female healthcare professionals.

Keywords: Burnout, professional. Health personnel. Occupational health. 
Transtornos mentais são caracterizados por sintomas de ansiedade, dificuldade de memória e concentração, fadiga, irritabilidade, insônia e queixas somáticas ${ }^{1}$. Seu desenvolvimento está relacionado a sofrimento psíquico, e costumam ser diagnosticados como ansiedade e depressão ${ }^{1,2}$. 0 índice de incidência desses quadros é significativo, sendo de $28,8 \%$ ao longo da vida para transtornos de ansiedade $^{3}$ e de 15 a $18 \%$ ao longo da vida em casos de depressão maior ${ }^{4}$. Pesquisas indicam correlação entre sintomas psíquicos e sofrimento relacionado ao trabalho, existindo três principais modelos conceituais: o modelo estresse-adaptação, o modelo demanda-controle e o burnout ${ }^{5}$.

Define-se "estresse" como a resposta do indivíduo a agente estressor que aciona a reação luta e fuga na tentativa de retornar ao estado de equilíbrio. Agentes estressores interferem no equilíbrio homeostático do organismo e podem ser físicos (originários do ambiente externo), cognitivos (avaliados como ameaças à integridade do indivíduo) ou emocionais (sentimentos ou acontecimentos com predominância do componente afetivo). As consequências desse acionamento são físicas e psicológicas, como aceleração do pensamento, aumento da função cardiorrespiratória e do tônus muscular e alteração da atenção ${ }^{6}$. O conceito de estresse é usado em medicina para nomear o conjunto de reações a situação que exige esforço adaptativo ${ }^{5}$.

O modelo estresse-adaptação indica que atualmente o estresse e a resposta adaptativa dos indivíduos são bem maiores que anteriormente, pois há cada vez mais pressões externas no trabalho rápidas mudanças tecnológicas, competitividade, pressão por resultados, recessão, medo de desemprego etc. ${ }^{5} \mathrm{O}$ modelo demanda-controle, por sua vez, associa a demanda psicológica de trabalho com o grau de autonomia e controle sobre a atividade laboral ${ }^{7}$, em que atividades com maior demanda psicológica e menor autonomia teriam maior potencial de causar doenças. Já o modelo do burnout é definido como resposta aos estressores interpessoais ocorridos na situação de trabalho ${ }^{8}$, sendo a síndrome de burnout a cronificação do estresse ocupacional.

O estresse ocupacional, diferente do estresse comum, tem o trabalho como fator essencial para seu desenvolvimento, ocorrendo quando não é possível para o trabalhador agir sobre os agentes causadores de estresse. Assim, o mecanismo de adaptação é rompido, persistem os sintomas de estresse e o organismo é deteriorado ou esgotado.

Comumente se descreve que a síndrome de burnout afeta profissionais que desempenham atividades com muito contato com outras pessoas, embora essa particularidade seja controversa ${ }^{5}$. Essa definição, contudo, gera diferentes termos para essa síndrome, como "estresse laboral", "profissional", "assistencial" ou "ocupacional", com termos ligados a percepções de adoecimento induzido pelo atendimento direto ao público. "Neurose profissional" ou "de excelência", "síndrome do esgotamento profissional" e "de queimar-se pelo trabalho" também são nomes utilizados. Essa variedade de nomenclaturas dificulta o levantamento bibliográfico na área ${ }^{9,10}$.

Os primeiros estudos sobre a síndrome surgiram na década de 1960, tornando-se mais numerosos e reconhecidos no Brasil - onde é identificada como doença relacionada ao trabalho ${ }^{11}$ - na década de $1970^{\circ}$. De acordo com Fabichak, Silva-Junior e Morrone ${ }^{12}$, estudos internacionais apresentam incidência de 50 a 74\% da síndrome de burnout em professores médicos, enfermeiros e residentes. No Brasil, os mesmos autores apontam que essa enfermidade acomete $78,4 \%$ dos residentes médicos de várias especialidades ${ }^{12}$.

O Maslach Burnout Inventory (MBI) é o instrumento mais utilizado para medir burnout ${ }^{13}$ e visa detectar a síndrome ou seu risco pela identificação de suas consequências. Consiste em 15 questões subdivididas em três subgrupos: exaustão emocional, tida como defasagem de energia e sentimento de esgotamento emocional; descrença ou despersonalização, indicada como falta de sensibilidade e rudeza ao tratar o público atendido; e eficácia profissional, definida como autoavaliação negativa do trabalhador ou redução dos sentimentos de competência no que se refere aos ganhos pessoais conquistados no trabalho ${ }^{14}$. As respostas variam de "nunca" a "todos os dias", e sua frequência é quantificada. Média de resultados elevada para exaustão emocional e descrença ou despersonalização e baixa para eficácia profissional indicaria síndrome de burnout.

Não há dados precisos sobre a incidência de síndrome de burnout, mas estima-se que varie de aproximadamente 4 a $85,7 \%$, a depender da população estudada ${ }^{15}$. No Brasil não há ainda muitas publicações sobre o tema ${ }^{15}$, o que aponta a 
importância da presente pesquisa, tendo em vista o impacto do adoecimento de profissionais da saúde sobre o bem-estar das pessoas atendidas, trazendo consequências sociais, pessoais e institucionais. Com isso, este estudo realizou revisão integrativa sobre a produção brasileira acerca do sofrimento psíquico na síndrome de burnout em profissionais da saúde entre 2014 e 2019.

\section{Método}

Trata-se de revisão integrativa da literatura estruturada nas seguintes etapas: definição da questão norteadora; busca em bancos de dados; coleta de informações; categorização e análise crítica dos estudos incluídos; discussão; e conclusão ${ }^{16}$. Elaborou-se a seguinte questão: qual foi o conhecimento produzido na literatura brasileira

Figura 1. Fluxograma da seleção dos artigos

\section{PubMed}

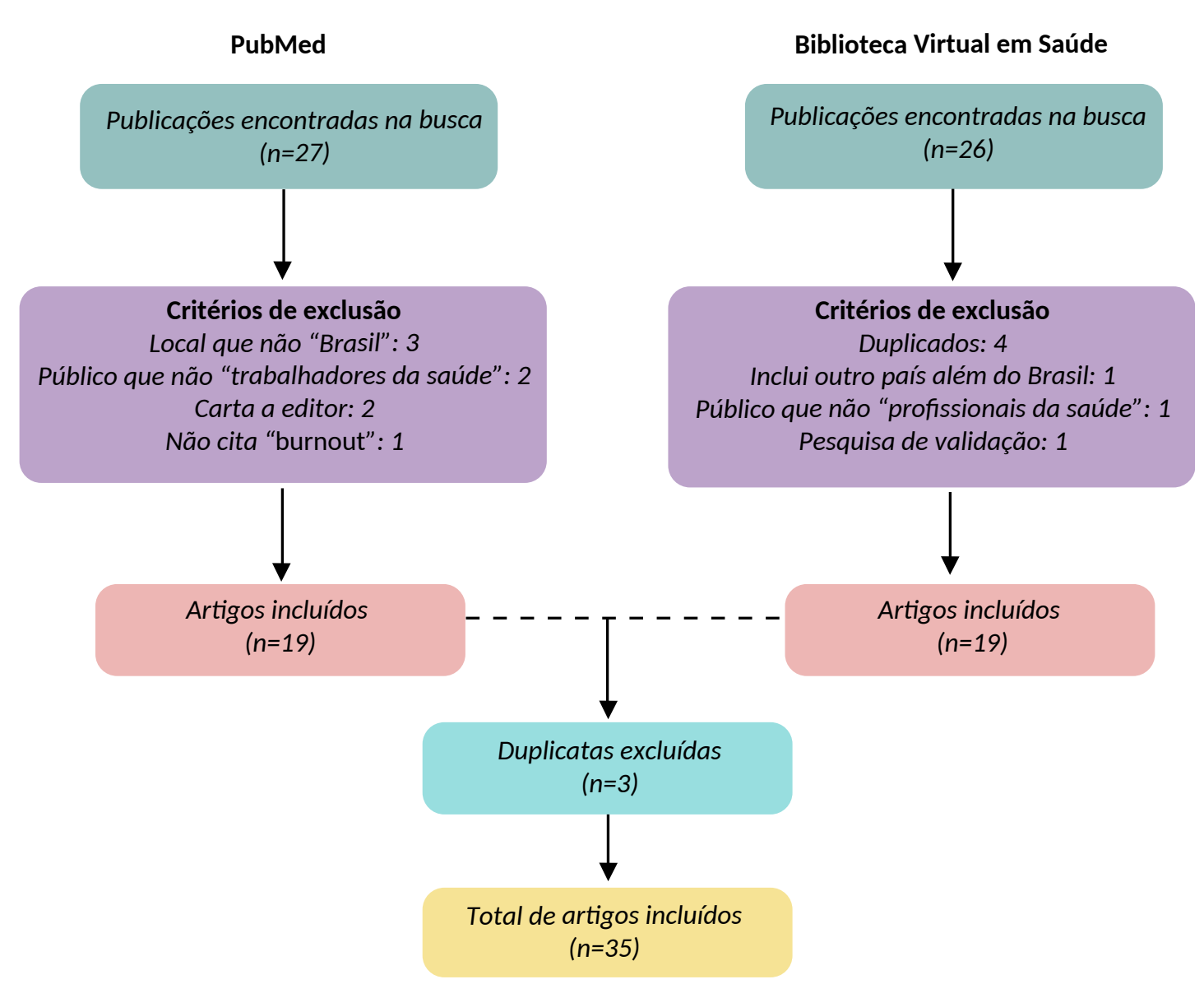

entre 2014 e 2019 sobre síndrome de burnout e trabalhadores da saúde?

Foram consultadas duas bases de dados. A pesquisa no PubMed envolveu os descritores "burnout" e "health personnel", extraídos do Medical Subject Headings. Na Biblioteca Virtual em Saúde foram utilizadas as palavras-chave "burnout" e "pessoal de saúde", extraídas dos Descritores em Ciências da Saúde. Em ambos os casos os descritores foram usados em associação com o operador booleano "and".

Os critérios de inclusão abrangeram artigos originais, dissertações e revisões de literatura publicados em português ou inglês (versões traduzidas de artigos brasileiros) entre 2014 e 2019, que delimitassem o Brasil como cenário de estudo e cuja amostra envolvesse trabalhadores da saúde. Foram excluídas as publicações que não atendessem aos critérios de inclusão, assim como duplicatas e trabalhos em outros formatos (Figura 1). 
Os dados foram coletados de forma independente pelas duas autoras e os resultados foram comparados para obter maior fidedignidade. Não foi utilizada ferramenta para selecionar e extrair dados. Após essa etapa, o corpus foi classificado de acordo com tipo de estudo, instrumentos utilizados, tamanho da amostra, local de pesquisa e formação dos autores (Tabela 1).

Tabela 1. Categorização dos artigos selecionados

\begin{tabular}{|c|c|c|c|c|}
\hline Autoria e ano & Tipo de estudo & Instrumentos utilizados & $\begin{array}{c}\text { Tamanho da amostra e local da } \\
\text { pesquisa }\end{array}$ & $\begin{array}{l}\text { Formação dos } \\
\text { autores }\end{array}$ \\
\hline $\begin{array}{l}\text { Almeida e } \\
\text { colaboradores; } \\
2016^{17}\end{array}$ & Revisão integrativa & $\begin{array}{l}\text { Critérios de seleção e } \\
\text { exclusão }\end{array}$ & $\begin{array}{l}\text { Oito artigos em biblioteca virtual } \\
\text { do Centro Latino-Americano e } \\
\text { do Caribe de Informação em } \\
\text { Ciências da Saúde }\end{array}$ & Enfermagem \\
\hline $\begin{array}{l}\text { Alves e } \\
\text { colaboradores; } \\
2018^{18}\end{array}$ & $\begin{array}{l}\text { Descritivo, } \\
\text { analítico e } \\
\text { exploratório }\end{array}$ & $\begin{array}{l}\text { Escala de Avaliação do } \\
\text { Impacto do Trabalho em } \\
\text { Serviços de Saúde Mental }\end{array}$ & $\begin{array}{l}70 \text { profissionais da enfermagem } \\
\text { em serviços psiquiátricos } \\
\text { hospitalares }\end{array}$ & $\begin{array}{l}\text { Enfermagem } \\
\text { Farmácia }\end{array}$ \\
\hline $\begin{array}{l}\text { Atanes e } \\
\text { colaboradores; } \\
2015^{19}\end{array}$ & $\begin{array}{l}\text { Estudo } \\
\text { correlacional de } \\
\text { corte transversal }\end{array}$ & $\begin{array}{l}\text { Escala de Conscientização } \\
\text { de Awareness, Escala de } \\
\text { Estresse Percebido, Escala } \\
\text { de Bem-Estar Subjetivo }\end{array}$ & $\begin{array}{l}\text { Médicos, enfermeiros, } \\
\text { auxiliares de enfermagem e } \\
\text { agentes comunitários de saúde } \\
\text { em UBS com ESF }\end{array}$ & $\begin{array}{l}\text { Medicina } \\
\text { Psicologia }\end{array}$ \\
\hline $\begin{array}{l}\text { Dorigan, } \\
\text { Guirardello; } \\
2018^{20}\end{array}$ & $\begin{array}{l}\text { Estudo } \\
\text { quantitativo } \\
\text { correlacional }\end{array}$ & $\begin{array}{l}\text { MBI, NWI-R, SAQ (short } \\
\text { form 2006) }\end{array}$ & $\begin{array}{l}465 \text { enfermeiros com registro } \\
\text { ativo no Conselho Regional de } \\
\text { Enfermagem de São Paulo e que } \\
\text { trabalham na assistência }\end{array}$ & Enfermagem \\
\hline $\begin{array}{l}\text { Fernandes, } \\
\text { Nitsche, Godoy; } \\
2018^{21}\end{array}$ & Quantitativa & $\begin{array}{l}\text { MBI, Questionário } \\
\text { Estruturado com História } \\
\text { Tabágica, Audit, Questionário } \\
\text { de Dependência de } \\
\text { Fagerström, mensuração do } \\
\text { monóxido de carbono }\end{array}$ & $\begin{array}{l}160 \text { profissionais de } \\
\text { enfermagem em quatro UTI de } \\
\text { hospital universitário }\end{array}$ & Enfermagem \\
\hline $\begin{array}{l}\text { Ferreira e } \\
\text { colaboradores; } \\
2017^{22}\end{array}$ & $\begin{array}{l}\text { Qualitativa } \\
\text { descritiva }\end{array}$ & Entrevistas & $\begin{array}{l}15 \text { enfermeiros e técnicos de } \\
\text { enfermagem em unidade de } \\
\text { saúde da família }\end{array}$ & Enfermagem \\
\hline $\begin{array}{l}\text { Garcia e } \\
\text { colaboradores; } \\
2017^{23}\end{array}$ & $\begin{array}{l}\text { Estudo } \\
\text { correlacional } \\
\text { transversal }\end{array}$ & $\begin{array}{l}\text { Caracterização profissional, } \\
\text { Instrumento Brasileiro } \\
\text { de Avaliação da Cultura } \\
\text { Organizacional e Escala de } \\
\text { Prazer e Sofrimento } \\
\text { no Trabalho }\end{array}$ & $\begin{array}{l}214 \text { membros da equipe de } \\
\text { enfermagem em hospital }\end{array}$ & Enfermagem \\
\hline $\begin{array}{l}\text { Garcia, Marziale; } \\
2018^{24}\end{array}$ & Revisão integrativa & $\begin{array}{l}\text { Busca manual com } \\
\text { descritores em bases de } \\
\text { dados }\end{array}$ & 14 artigos & Enfermagem \\
\hline $\begin{array}{l}\text { Guirardello; } \\
2017^{25}\end{array}$ & Estudo transversal & $\begin{array}{l}\text { MBI, NWI-R, Questionário } \\
\text { de Atitudes de Segurança }\end{array}$ & $\begin{array}{l}114 \text { profissionais de } \\
\text { enfermagem em UTI em } \\
\text { hospital de ensino }\end{array}$ & Enfermagem \\
\hline $\begin{array}{l}\text { Hoppen e } \\
\text { colaboradores; } \\
2017^{26}\end{array}$ & Estudo transversal & $\mathrm{MBI}$ & 52 médicos em UTI & Medicina \\
\hline $\begin{array}{l}\text { Leite, } \\
\text { Nascimento, } \\
\text { Oliveira; } 2014^{27}\end{array}$ & $\begin{array}{l}\text { Estudo qualitativo, } \\
\text { descritivo e } \\
\text { exploratório }\end{array}$ & $\begin{array}{l}\text { Entrevistas para avaliar } \\
\text { percepção de qualidade } \\
\text { de vida }\end{array}$ & $\begin{array}{l}40 \text { profissionais de saúde em } \\
\text { cinco UBS com ESF }\end{array}$ & $\begin{array}{l}\text { Enfermagem } \\
\text { Fisioterapia }\end{array}$ \\
\hline
\end{tabular}


Tabela 1. Continuação

\begin{tabular}{|c|c|c|c|c|}
\hline Autoria e ano & Tipo de estudo & Instrumentos utilizados & $\begin{array}{c}\text { Tamanho da amostra e local da } \\
\text { pesquisa }\end{array}$ & $\begin{array}{l}\text { Formação dos } \\
\text { autores }\end{array}$ \\
\hline $\begin{array}{l}\text { Lorenz, Sabino, } \\
\text { Corrêa Filho; } \\
2018^{28}\end{array}$ & $\begin{array}{l}\text { Estudo transversal } \\
\text { e correlacional }\end{array}$ & $\begin{array}{l}\text { MBI, questionário para } \\
\text { caracterizar os enfermeiros, } \\
\text { para avaliar percepção } \\
\text { sobre a qualidade do } \\
\text { cuidado e de recursos } \\
\text { materiais e humanos e } \\
\text { para verificar intenções de } \\
\text { deixar o trabalho atual e a } \\
\text { enfermagem }\end{array}$ & $\begin{array}{l}198 \text { enfermeiros em UBS com } \\
\text { ESF em Campinas/SP }\end{array}$ & $\begin{array}{l}\text { Enfermagem } \\
\text { Medicina }\end{array}$ \\
\hline $\begin{array}{l}\text { Lorenz, } \\
\text { Guirardello; } \\
2014^{29}\end{array}$ & $\begin{array}{l}\text { Estudo transversal } \\
\text { e correlacional }\end{array}$ & $\begin{array}{l}\text { MBI, NWI-R, ficha } \\
\text { de caracterização do } \\
\text { enfermeiro }\end{array}$ & $\begin{array}{l}198 \text { enfermeiros em UBS de um } \\
\text { município brasileiro }\end{array}$ & Enfermagem \\
\hline $\begin{array}{l}\text { Martins e } \\
\text { colaboradores; } \\
2014^{30}\end{array}$ & $\begin{array}{l}\text { Exploratória, } \\
\text { descritiva e } \\
\text { correlacional }\end{array}$ & $\begin{array}{l}\text { MBI, questionário com } \\
\text { aspectos individuais, } \\
\text { sociodemográficos e área } \\
\text { de cobertura da equipe }\end{array}$ & $\begin{array}{l}107 \text { trabalhadores da atenção } \\
\text { primária à saúde de três } \\
\text { municípios de pequeno porte } \\
\text { de Minas Gerais }\end{array}$ & Psicologia \\
\hline $\begin{array}{l}\text { Mattos, Araújo, } \\
\text { Almeida; } 2017^{31}\end{array}$ & Estudo transversal & $\begin{array}{l}\text { Self Reporting } \\
\text { Questionnaire-20 }\end{array}$ & $\begin{array}{l}2.523 \text { trabalhadores da atenção } \\
\text { básica em cinco cidades da Bahia }\end{array}$ & $\begin{array}{l}\text { Enfermagem } \\
\text { Psicologia }\end{array}$ \\
\hline $\begin{array}{l}\text { Maissiat e } \\
\text { colaboradores; } \\
2015^{32}\end{array}$ & Estudo transversal & $\begin{array}{l}\text { Escala de Avaliação do } \\
\text { Contexto de Trabalho e Escala } \\
\text { de Indicadores de Prazer e } \\
\text { Sofrimento no Trabalho }\end{array}$ & $\begin{array}{l}242 \text { trabalhadores da atenção } \\
\text { básica em } 15 \text { unidades de saúde } \\
\text { de um município do Rio Grande } \\
\text { do Sul }\end{array}$ & Enfermagem \\
\hline $\begin{array}{l}\text { Migowski, Piccoli, } \\
\text { Quevedo; } 2016^{33}\end{array}$ & $\begin{array}{l}\text { Estudo descritivo, } \\
\text { de corte } \\
\text { transversal }\end{array}$ & $\begin{array}{l}\text { Quality of Working Life } \\
\text { Questionnaire-78 }\end{array}$ & $\begin{array}{l}95 \text { enfermeiros e técnicos de } \\
\text { enfermagem em hospital da } \\
\text { Serra Gaúcha, Rio Grande do Sul }\end{array}$ & $\begin{array}{l}\text { Enfermagem } \\
\text { Matemática } \\
\text { Educação } \\
\text { Física }\end{array}$ \\
\hline $\begin{array}{l}\text { Mota, Dosea, } \\
\text { Nunes; } 2014^{34}\end{array}$ & $\begin{array}{l}\text { Estudo transversal } \\
\text { e quantitativo }\end{array}$ & $\begin{array}{l}\text { MBI, Job Stress } \\
\text { Scale, questionário } \\
\text { socioeconômico e } \\
\text { ocupacional }\end{array}$ & $\begin{array}{l}222 \text { agentes comunitários de } \\
\text { saúde em } 43 \text { unidades de saúde } \\
\text { da família de Aracaju/SE }\end{array}$ & Fisioterapia \\
\hline $\begin{array}{l}\text { Oliveira e } \\
\text { colaboradores; } \\
2018^{35}\end{array}$ & Estudo transversal & $\begin{array}{l}\text { MBI, Job Satisfaction } \\
\text { Survey, PHQ-9, SAQ }\end{array}$ & $\begin{array}{l}271 \text { trabalhadores (incluindo } \\
\text { pessoal de apoio) em hospital de } \\
\text { ensino no estado de São Paulo }\end{array}$ & Farmácia \\
\hline $\begin{array}{l}\text { Dal Pai e } \\
\text { colaboradores; } \\
2015^{36}\end{array}$ & Estudo transversal & $\begin{array}{l}\text { MBI, Survey Questionnaire: } \\
\text { Workplace Violence no } \\
\text { Setor da Saúde, Self-Report } \\
\text { Questionnaire }\end{array}$ & $\begin{array}{l}269 \text { profissionais em hospital } \\
\text { público }\end{array}$ & Enfermagem \\
\hline $\begin{array}{l}\text { Pegoraro, } \\
\text { Schaefer, Zoboli; } \\
2017^{37}\end{array}$ & $\begin{array}{l}\text { Revisão de } \\
\text { literatura }\end{array}$ & $\begin{array}{l}\text { Busca manual com } \\
\text { descritores em bases } \\
\text { de dados }\end{array}$ & 35 artigos & Enfermagem \\
\hline $\begin{array}{l}\text { Pereira-Lima, } \\
\text { Loureiro, Crippa; } \\
2016^{38}\end{array}$ & $\begin{array}{l}\text { Pesquisa } \\
\text { quantitativa }\end{array}$ & $\begin{array}{l}\text { PHQ-4, Audit-3, } \\
\text { NEO Five Factor } \\
\text { Inventory, Social Skills } \\
\text { Inventory, questionário } \\
\text { sociodemográfico }\end{array}$ & $\begin{array}{l}270 \text { médicos residentes de } \\
\text { hospital em Ribeirão Preto/SP }\end{array}$ & $\begin{array}{l}\text { Medicina } \\
\text { Psicologia }\end{array}$ \\
\hline $\begin{array}{l}\text { Portela e } \\
\text { colaboradores; } \\
2015^{39}\end{array}$ & Revisão integrativa & $\begin{array}{l}\text { Busca manual com } \\
\text { descritores em bases } \\
\text { de dados }\end{array}$ & $\begin{array}{l}11 \text { artigos em cinco bases de } \\
\text { dados }\end{array}$ & Enfermagem \\
\hline
\end{tabular}

continua... 
Tabela 1. Continuação

\begin{tabular}{|c|c|c|c|c|}
\hline Autoria e ano & Tipo de estudo & Instrumentos utilizados & $\begin{array}{c}\text { Tamanho da amostra e local da } \\
\text { pesquisa }\end{array}$ & $\begin{array}{l}\text { Formação dos } \\
\text { autores }\end{array}$ \\
\hline $\begin{array}{l}\text { Santos, Neri, } \\
\text { Wanderley; } \\
2018^{40}\end{array}$ & $\begin{array}{l}\text { Pesquisa } \\
\text { quantitativa }\end{array}$ & $\mathrm{MBI}$ & $\begin{array}{l}48 \text { fisioterapeutas em hospital, } \\
\text { a maioria em UTI }\end{array}$ & Fisioterapia \\
\hline $\begin{array}{l}\text { Silva e } \\
\text { colaboradores; } \\
2015^{41}\end{array}$ & $\begin{array}{l}\text { Estudo descritivo } \\
\text { seccional }\end{array}$ & $\begin{array}{l}\text { MBI e Self Report } \\
\text { Questionnaire para avaliar } \\
\text { transtornos mentais } \\
\text { comuns }\end{array}$ & $\begin{array}{l}130 \text { enfermeiros, técnicos e } \\
\text { auxiliares de enfermagem em } \\
\text { UTI e unidade coronariana de } \\
\text { dois hospitais de grande porte } \\
\text { na cidade do Rio de Janeiro/RJ }\end{array}$ & $\begin{array}{l}\text { Enfermagem } \\
\text { Biologia }\end{array}$ \\
\hline Silva; $2015^{42}$ & Estudo transversal & MBI, PHQ-9 & $\begin{array}{l}2.940 \text { trabalhadores de saúde } \\
\text { em ESF de Pandora/SP }\end{array}$ & Medicina \\
\hline $\begin{array}{l}\text { Silva e } \\
\text { colaboradores; } \\
2015^{43}\end{array}$ & Estudo transversal & $\begin{array}{l}\text { MBI, questionário } \\
\text { sociodemográfico }\end{array}$ & $\begin{array}{l}198 \text { profissionais de saúde } \\
\text { com nível superior em Rede de } \\
\text { Atenção Primária à Saúde do } \\
\text { município de Aracaju/SE }\end{array}$ & Medicina \\
\hline $\begin{array}{l}\text { Silveira e } \\
\text { colaboradores; } \\
2016^{44}\end{array}$ & $\begin{array}{l}\text { Revisão } \\
\text { sistemática }\end{array}$ & $\begin{array}{l}\text { Busca manual com } \\
\text { descritores em bases de } \\
\text { dados }\end{array}$ & $\begin{array}{l}17 \text { artigos nas bases de dados } \\
\text { Medical Literature Analysis } \\
\text { and Retrieval System Online, } \\
\text { Literatura Latino-Americana e do } \\
\text { Caribe em Ciências da Saúde e } \\
\text { Scientific Electronic Library Online }\end{array}$ & Medicina \\
\hline Souza; $2017^{45}$ & Estudo transversal & $\begin{array}{l}\text { Versão abreviada do World } \\
\text { Health Organization Quality } \\
\text { of Life-100, questionário } \\
\text { sociodemográfico }\end{array}$ & $\begin{array}{l}664 \text { profissionais de } \\
\text { enfermagem de unidades } \\
\text { pediátricas hospitalares de } \\
\text { três hospitais-escola de Belo } \\
\text { Horizonte/MG }\end{array}$ & Enfermagem \\
\hline $\begin{array}{l}\text { Tironi e } \\
\text { colaboradores; } \\
2016^{46}\end{array}$ & $\begin{array}{l}\text { Estudo } \\
\text { epidemiológico } \\
\text { descritivo }\end{array}$ & $\begin{array}{l}\text { MBI, questionário } \\
\text { sociodemográfico }\end{array}$ & $\begin{array}{l}180 \text { médicos intensivistas em } \\
\text { cinco capitais (Porto Alegre/ } \\
\text { RS, São Paulo/SP, Salvador/BA, } \\
\text { Goiânia/GO e Belém/PA) }\end{array}$ & $\begin{array}{l}\text { Enfermagem } \\
\text { Medicina } \\
\text { Psicologia } \\
\text { Geografia }\end{array}$ \\
\hline $\begin{array}{l}\text { Vasconcelos, } \\
\text { Martino, França; } \\
2018^{47}\end{array}$ & $\begin{array}{l}\text { Estudo } \\
\text { quantitativo, } \\
\text { descritivo, } \\
\text { transversal }\end{array}$ & $\begin{array}{l}\text { MBI, questionário } \\
\text { sociodemográfico, } \\
\text { Inventário de Depressão de } \\
\text { Beck (versão 1) }\end{array}$ & $\begin{array}{l}91 \text { enfermeiros em UTI de } \\
\text { hospital universitário da cidade } \\
\text { de São Paulo/SP }\end{array}$ & Enfermagem \\
\hline $\begin{array}{l}\text { Vidotti e } \\
\text { colaboradores; } \\
2018^{48}\end{array}$ & Estudo transversal & $\begin{array}{l}\text { MBI, Demand-Control- } \\
\text { Support Questionnaire }\end{array}$ & $\begin{array}{l}502 \text { trabalhadores de } \\
\text { enfermagem em instituição } \\
\text { hospitalar filantrópica }\end{array}$ & Enfermagem \\
\hline Zampieri; $2016^{49}$ & Editorial & - & UTI & Medicina \\
\hline $\begin{array}{l}\text { Zanatta, Lucca; } \\
2015^{50}\end{array}$ & $\begin{array}{l}\text { Estudo exploratório, } \\
\text { descritivo, com } \\
\text { delineamento } \\
\text { transversal e } \\
\text { abordagem } \\
\text { quantitativa }\end{array}$ & $\begin{array}{l}\text { MBI, formulário de } \\
\text { dados biossociais, roteiro } \\
\text { de observação não } \\
\text { participante }\end{array}$ & $\begin{array}{l}188 \text { médicos, enfermeiros e } \\
\text { técnicos de enfermagem em } \\
1 \text { hospital onco-hematológico } \\
\text { infantil no estado de São Paulo }\end{array}$ & $\begin{array}{l}\text { Enfermagem } \\
\text { Medicina }\end{array}$ \\
\hline $\begin{array}{l}\text { Zavalis e } \\
\text { colaboradores; } \\
2015^{51}\end{array}$ & $\begin{array}{l}\text { Estudo } \\
\text { quantitativo, } \\
\text { correlacional } \\
\text { descritivo }\end{array}$ & $\begin{array}{l}\text { Instrumento de coleta de } \\
\text { dados baseado em fatores } \\
\text { estressores, questionário } \\
\text { sociodemográfico }\end{array}$ & $\begin{array}{l}50 \text { profissionais de enfermagem } \\
\text { da assistência de hospital do Rio } \\
\text { de Janeiro/RJ }\end{array}$ & $\begin{array}{l}\text { Enfermagem } \\
\text { Nutrição } \\
\text { Estatística }\end{array}$ \\
\hline
\end{tabular}

Audit: Alcohol Use Disorders Identification Test; ESF: Estratégia Saúde da Familia; MBI: Maslach Burnout Inventory; NWI-R: Nursing Work Index-Revised; PHQ: Patient Health Questionnaire; SAQ: Safety Attitudes Questionnaire; UBS: unidades básicas de saúde; UTI: unidade de terapia intensiva 


\section{Resultados e discussão}

Segundo os resultados, muitos estudos não utilizaram instrumentos que medem a síndrome de burnout, preferindo dispositivos voltados a outros aspectos da saúde do trabalhador 17-19,22-24,27,31-33,37-39,44,45,51. Esses trabaIhos foram, contudo, considerados nesta pesquisa porque se referem a sofrimento psíquico de trabalhadores da saúde, relacionando-o a burnout em seu conteúdo. Observa-se ainda que a maioria das pesquisas apontou predominância de profissionais de saúde do sexo feminino ${ }^{18,19-22,25,27-29,31,32,34,36,39,42,43,45-47,50,51}$, principalmente em enfermagem ${ }^{18,20-22,25,28,29,39,45,47,51}$.

Dos 35 artigos incluídos, 27 (77\%) empregaram abordagem quantitativa, 2 (6\%), qualitativa, 5 (14\%) eram revisão da literatura e 1 (3\%) era editorial. Dentre as pesquisas quantitativas, 18 (67\% deste subgrupo) aplicaram o instrumento $\mathrm{MBI}^{14}$, indicando ser este o mais utilizado para medir síndrome de burnout, o que corrobora as afirmações de Tamayo e Troccoli ${ }^{13}$. Argumenta-se que, por um lado, a ampla utilização do MBI é interessante para comparar resultados, mas, por outro, limita o entendimento da síndrome ao que é perguntado no instrumento ${ }^{13}$.

Dentre as pesquisas qualitativas e quantitativas, 18 (62\% deste subgrupo) foram realizadas em hospital ou tratavam desse ambiente, e 11 (38\%) focavam ou foram executadas no âmbito da atenção básica. Os resultados refletem a tradição de abordar o bem-estar do trabalhador da saúde, principalmente de enfermagem e medicina, majoritariamente em ambientes hospitalares, considerados os locais com maior risco ocupacional de provocar doenças mentais nessa população ${ }^{17}$.

O número significativo de pesquisas desenvolvidas na atenção básica provavelmente se deve ao protagonismo deste setor, cujos profissionais estão frequentemente sobrecarregados, desempenhando papéis que vão além das tarefas delimitadas pelo cargo, com destaque para a enfermagem ${ }^{52}$. É necessário investigar a síndrome de burnout em outros locais de trabalho, dado que ambulatórios, unidades de pronto atendimento, Centros de Atenção Psicossocial e outras unidades do Sistema Único de Saúde não foram abrangidos nos artigos encontrados nesta pesquisa.
A maioria dos autores da amostra era formada em enfermagem - também a principal profissão pesquisada -, com 24 (69\%) $\operatorname{artigos}^{17,18,20-25,27-29,31-33,36,37,39,41,45-48,50,51}$ contendo ao menos um pesquisador da categoria. Dez (29\%) trabalhos ${ }^{19,26,28,38,42-44,46,49,50}$ contemplavam ao menos um pesquisador médico, e apenas 13 (37\%) pesquisas ${ }^{18,19,27,30,31,33-35,38,40,41,46,51}$ incluíam autores de outras categorias profissionais. Isso provavelmente se deve à tradição de pesquisas em ambiente hospitalar ${ }^{17} \mathrm{e}$ ao fato de os profissionais que ali trabaIham serem em sua maioria enfermeiros e médicos, que por sua profissão estão expostos a fatores de estresse adicionais ${ }^{52}$. $\mathrm{Na}$ área da saúde, o trabalho do médico é tradicionalmente o mais estudado do ponto de vista do impacto psicológico ${ }^{5}$, mas pesquisas relatam especial risco de profissionais de enfermagem desenvolverem distúrbios decorrentes do estresse vivido no trabalho ${ }^{53}$.

Por sua definição, a síndrome de burnout é adoecimento relacionado ao trabalho ${ }^{54}$. Sendo assim, a maioria das pesquisas incluídas neste estudo aponta a importância de os gestores promoverem ações interventivas e de prevenção. Concluem também que é preciso diagnosticar a síndrome precocemente, que muitos profissionais apresentam risco elevado de desenvolvê-la, associado a alto risco de depressão, e que dificuldade nas relações hierárquicas e recursos físicos e humanos insuficientes são fatores estressantes, relacionando ainda fatores psicossociais e idade jovem. Recomendam também que sejam realizados mais estudos na área.

Dentre os 35 artigos da amostra, 29 (83\%) concluíram que as condições laborais estão relacionadas ao burnout e, dentre esses, 13 (45\%) sugerem diretamente a necessidade de a gestão intervir no ambiente de trabalho como medida de saúde. A literatura aponta que o tratamento de burnout deve considerar a origem da síndrome, abrangendo aspectos pessoais, laborais e de organização do trabalho ${ }^{55}$. Tratar apenas um de seus sintomas, como depressão ou ansiedade, seria paliativo, já que se trata de fenômeno coletivo e organizacional. Por isso é importante estudar a síndrome para melhor tratá-la6.

É possível relacionar a falta de autonomia no trabalho com burnout ou outro tipo de adoecimento mental, sendo associado à organização das tarefas. A autonomia, entendida como a possibilidade de manifestar desejo e subjetividade no 
trabalho ${ }^{56}$, permitiria ao trabalhador interferir naquilo que lhe causa sofrimento. Nesta situação, o adoecimento ocorre quando o trabalhador é forçado a ir sistematicamente além de seu limite subjetivo ${ }^{57}$. A alta demanda de trabalho relacionada a baixa autonomia trazem maior risco de burnout ${ }^{58}$. O sujeito não pode expressar os sentimentos mobilizados pelo sofrimento no trabalho, devendo suprimi-los, o que gera o processo que SeligmannSilva ${ }^{59}$ nomeou de "desgaste". Isso indica que as estratégias de intervenção podem incluir o aumento da autonomia dos profissionais ${ }^{25}$.

Por fim, conforme apresentado, a maioria dos estudos ${ }^{18,20-22,25,28,29,39,45,47,51}$ revelou predomínio do sexo feminino no exercício da enfermagem. Contudo, este dado não foi analisado em nenhuma das pesquisas selecionadas, evidenciando que o impacto do sexo no adoecimento e na dinâmica do trabalho tem sido negligenciado pelos pesquisadores. A associação entre a predominância do sexo feminino na enfermagem e o cuidado é histórica ${ }^{60}$ e carrega marcas culturais, impactando também as escolhas dos pesquisadores. Também já foi descrito na literatura o quanto a dupla jornada de trabalho das mulheres, a tendência cultural a não valorizar o trabalho feminino e a hegemonia do discurso médico impactam a saúde mental das profissionais de saúde ${ }^{5}$, sendo fatores de estresse além do desgaste profissional.

\section{Considerações finais}

A literatura sobre síndrome de burnout em profissionais da saúde ainda é escassa, possivelmente pelo fato de a Classificação Estatística Internacional de Doenças e Problemas Relacionados com a Saúde, tanto em sua versão $10^{61}$ quanto $11^{54}$, trazer o burnout não como condição de saúde, mas fenômeno ocupacional, o que pode resultar em subdiagnóstico. Apesar de os números serem incertos, dada a carência de pesquisas sobre o tema e a prevalência de amostras pequenas com baixo poder de generalização, as conclusões de muitos dos artigos encontrados nesta pesquisa apontam para índice significativo de síndrome de burnout em profissionais da saúde.

A queda na qualidade do trabalho, o aumento do absenteísmo, da rotatividade e do número de acidentes de trabalho prejudicam as empresas em termos financeiros e de imagem. Além disso, provocam prejuízo social pela diminuição do número de adultos em idade produtiva devido ao adoecimento e gastos com saúde. Os impactos como um todo são abrangentes: de ordem pessoal, social, empresarial, governamental e sobre o público atendido.

São necessárias pesquisas que considerem a prevalência do sexo feminino e seu impacto, bem como estudos que abranjam outras profissões além de enfermagem e medicina, uma vez que é preciso saber se de fato essas áreas são as mais atingidas por síndrome de burnout ou se seus índices parecem maiores por serem as mais pesquisadas. Também é necessário investigar ambientes além do hospital e da atenção básica. Com isso, os dados serão mais consistentes e instruirão intervenções mais eficazes nas instituições de saúde para combater e prevenir a síndrome de burnout.

\section{Referências}

1. Green MJ, Benzeval M. Ageing, social class and common mental disorders: longitudinal evidence from three cohorts in the West of Scotland. Psychol Med [Internet]. 2011 [acesso 16 set 2020];41(3):565-74. DOI: $10.1017 /$ S0033291710000851

2. Goldberg D, Huxley P. Common mental disorders: a bio-social model. London: Tavistock; 1992.

3. Kessler RC, Berglund P, Demler O, Jin R, Merikangas KR, Walters EE. Lifetime prevalence and age-of-onset distributions of DSM-IV disorders in the national comorbidity survey replication. Arch Gen Psychiatry [Internet]. 2005 [acesso 16 set 2020];62(6):593-602. DOI: 10.1001/archpsyc.62.6.593

4. Kessler RC, Birnbaum H, Bromet E, Hwang I, Sampson N, Shahly V. Age differences in major depression: results from the National Comorbidity Survey Replication (NCS-R). Psychol Med [Internet]. 2010 [acesso 16 set 2020];40(2):225-37. DOI: 10.1017/S0033291709990213 
5. Martins LAN. A saúde do profissional de saúde. In: Marco MA, organizador. A face humana da medicina: do modelo biomédico ao modelo biopsicossocial. São Paulo: Casa do Psicólogo; 2003. p. 92-7.

6. Benevides-Pereira AMT. Burnout: o processo de adoecer pelo trabalho. In: Benevides-Pereira AMT, organizadora. Burnout: quando o trabalho ameaça o bem-estar do trabalhador. $4^{a}$ ed. São Paulo: Casa do Psicólogo; 2010. p. 21-91.

7. Karasek RA Jr. Job demands, job decision latitude, and mental strain: implications for job redesign. Adm Sci Q [Internet]. 1979 [acesso 16 set 2020];24(2):285-308. DOI: 10.2307/2392498

8. Benevides-Pereira AMT. Burnout, por quê? In: Benevides-Pereira AMT, organizadora. Op. cit. p. 13-20. p. 15.

9. Borges LO, Argolo JCT, Pereira ALS, Machado EAP, Silva WS. A síndrome de burnout e os valores organizacionais: um estudo comparativo em hospitais universitários. Psicol Reflex Crít [Internet]. 2002 [acesso 16 set 2020];15(1):189-200. DOI: 10.1590/S0102-79722002000100020

10. Benevides-Pereira AMT. O estado da arte do burnout no Brasil. Rev Eletrônica InterAção Psy [Internet]. 2003 [acesso 16 set 2020];1(1):4-11. Disponível: https://bit.ly/2YxBivs

11. Brasil. Ministério da Saúde, Organização Pan-Americana da Saúde. Doenças relacionadas ao trabalho: manual de procedimentos para os serviços de saúde. Brasília: Ministério da Saúde; 2001. Disponível: https://bit.ly/3tg4Z2h

12. Fabichak C, Silva-Junior JS, Morrone LC. Síndrome de burnout em médicos residentes e preditores organizacionais do trabalho. Rev Bras Med Trab [Internet]. 2014 [acesso 16 set 2020];12(2):79-84. Disponível: https://bit.ly/3j8nkKa

13. Tamayo MR, Troccoli BT. Construção e validação fatorial da Escala de Caracterização do Burnout (ECB). Estud Psicol [Internet]. 2009 [acesso 16 set 2020];14(3):213-21. DOI: 10.1590/S1413-294X2009000300005

14. Maslach C, Schaufeli WB, Leiter MP. Job burnout. Annu Rev Psychol [Internet]. 2001 [acesso 16 set 2020];52:397-422. DOI: 10.1146/annurev.psych.52.1.397

15. Trigo TR, Teng CT, Hallar JEC. Síndrome de burnout ou estafa profissional e os transtornos psiquiátricos. Rev Psiquiatr Clín [Internet]. 2007 [acesso 16 set 2020];34(5):223-33. DOI: 10.1590/S0101-60832007000500004

16. Ercole FF, Melo LS, Alcoforado CLG. Revisão integrativa versus revisão sistemática. Reme [Internet]. 2014 [acesso 16 set 2020];18(1):9-11. DOI: 10.5935/1415-2762.20140001

17. Almeida LA, Medeiros IDS, Barros AG, Martins CCF, Santos VEP. Fatores geradores da síndrome de burnout em profissionais da saúde. Rev Pesqui [Internet]. 2016 [acesso 31 jul 2019];8(3):4623-8. DOI: 10.9789/ 2175-5361.2016.v8i3.4623-4628

18. Alves SR, Santos RP, Oliveira RG, Yamaguchi MU. Serviços de saúde mental: percepção da enfermagem em relação à sobrecarga e condições de trabalho. Rev Pesqui [Internet]. 2018 [acesso 18 out 2019];10(1):25-9. DOI: 10.9789/2175-5361.2018.v10i1.25-29

19. Atanes ACM, Andreoni S, Hirayama MS, Montero-Marin J, Barros VV, Ronzani TM et al. Mindfulness, perceived stress, and subjective well-being: a correlational study in primary care health professionals. BMC Complement Altern Med [Internet]. 2015 [acesso 16 set 2020];15:303. DOI: 10.1186/s12906-015-0823-0

20. Dorigan GH, Guirardello EB. Efeito do ambiente da prática do enfermeiro nos resultados do trabalho e clima de segurança. Rev Latinoam Enferm [Internet]. 2018 [acesso 31 jul 2019];26:e3056. DOI: 10.1590/ 1518-8345.2633.3056

21. Fernandes LS, Nitsche MJT, Godoy I. Associação entre síndrome de burnout, uso prejudicial de álcool e tabagismo na enfermagem nas UTIs de um hospital universitário. Ciênc Saúde Coletiva [Internet]. 2018 [acesso 14 maio 2019];23(1):203-14. DOI: 10.1590/1413-81232018231.05612015

22. Ferreira JS, Ribeiro KV, Caramuru PS, Hanzelmann RS, Velasco AR, Passos JP. Estresse e estratégias de enfrentamento em trabalhadores de enfermagem de uma unidade de saúde da família. Rev Pesqui [Internet]. 2017 [acesso 14 maio 2019];9(3):818-23. DOI: 10.9789/2175-5361.2017.v9i3.818-823

23. Garcia AB, Rocha FLR, Pissinati PSC, Marziale MHP, Camelo SHH, Haddad MCFL. The effects of organizational culture on nurses' perceptions of their work. Br J Nurs [Internet]. 2017 [acesso 31 jul 2019];26(14):806-12. DOI: 10.12968/bjon.2017.26.14.806 
24. Garcia GPA, Marziale MHP. Indicadores de esgotamento profissional em trabalhadores da atenção primária à saúde. Rev Bras Enferm [Internet]. 2018 [acesso 31 jul 2019];71(supl 5):2334-42. DOI: 10.1590/ 0034-7167-2017-0530

25. Guirardello EB. Impacto do ambiente de cuidados críticos no burnout, percepção da qualidade do cuidado e atitude de segurança da equipe de enfermagem. Rev Latinoam Enferm [Internet]. 2017 [acesso 14 maio 2019];25:e2884. DOI: 10.1590/1518-8345.1472.2884

26. Hoppen CMS, Kissmann N, Chinelato JR, Coelho VP, Wenczenovicz C, Nunes FCL, Friedman G. Alta prevalência de síndrome de burnout em médicos intensivistas da cidade de Porto Alegre. Rev Bras Ter Intensiva [Internet]. 2017 [acesso 16 set 2020];29(1):115-20. DOI: 10.5935/0103-507X.20170017

27. Leite DF, Nascimento DDG, Oliveira MAC. Qualidade de vida no trabalho de profissionais do NASF no município de São Paulo. Physis [Internet]. 2014 [acesso 14 maio 2019];24(2):507-25. DOI: 10.1590/ S0103-73312014000200010

28. Lorenz VR, Sabino MO, Corrêa Filho HR. Esgotamento profissional, qualidade e intenções entre enfermeiros de saúde da família. Rev Bras Enferm [Internet]. 2018 [acesso 14 maio 2019];71(supl 5):2295-301. DOI: 10.1590/0034-7167-2016-0510

29. Lorenz VR, Guirardello EB. The environment of professional practice and burnout in nurses in primary healthcare. Rev Latinoam Enferm [Internet]. 2014 [acesso 14 maio 2019];22(6):926-33. DOI: 10.1590/ 0104-1169.0011.2497

30. Martins LF, Laport TJ, Menezes VP, Medeiros PB, Ronzani TM. Esgotamento entre profissionais da atenção primária à saúde. Ciênc Saúde Coletiva [Internet]. 2014 [acesso 14 maio 2019];19(12):4739-50. DOI: 10.1590/1413-812320141912.03202013

31. Mattos AIS, Araújo TM, Almeida MMG. Interaction between demand-control and social support in the occurrence of common mental disorders. Rev Saúde Pública [Internet]. 2017 [acesso 11 jul 2019];51:48. DOI: $10.1590 / \mathrm{s} 1518-8787.2017051006446$

32. Maissiat GS, Lautert L, Dal Pai D, Tavares JP. Contexto de trabalho, prazer e sofrimento na atenção básica em saúde. Rev Gaúcha Enferm [Internet]. 2015 [acesso 14 maio 2019];36(2):42-9. DOI: 10.1590/ 1983-1447.2015.02.51128

33. Migowski ER, Piccoli JCJ, Quevedo DM. Qualidade de vida no trabalho: percepção de enfermeiros e técnicos em enfermagem de um hospital da Serra Gaúcha, RS, Brasil. Mundo Saúde [Internet]. 2016 [acesso 16 set 2020];40(2):189-98. DOI: 10.15343/0104-7809.20164002189198

34. Mota CM, Dosea GS, Nunes PS. Avaliação da presença da síndrome de burnout em agentes comunitários de saúde no município de Aracaju, Sergipe, Brasil. Ciênc Saúde Coletiva [Internet]. 2014 [acesso 14 maio 2019];19(12):4719-26. DOI: 10.1590/1413-812320141912.02512013

35. Oliveira AM, Silva MT, Galvão TF, Lopes LC. The relationship between job satisfaction, burnout syndrome and depressive symptoms: an analysis of professionals in a teaching hospital in Brazil. Medicine (Baltimore) [Internet]. 2018 [acesso 16 set 2020];97(49):e13364. DOI: 10.1097/MD.0000000000013364

36. Dal Pai D, Lautert L, Souza SBC, Marziale MHP, Tavares JP. Violência, burnout e transtornos psíquicos menores no trabalho hospitalar. Rev Esc Enferm USP [Internet]. 2015 [acesso 16 set 2020];49(3):457-64. DOI: 10.1590/S0080-623420150000300014

37. Pegoraro PBB, Schaefer R, Zoboli ELCP. Desgaste psíquico-moral nos trabalhadores da atenção primária. Rev Esc Enferm USP [Internet]. 2017 [acesso 31 jul 2019];51:e03257. DOI: 10.1590/s1980-220x2016035203257

38. Pereira-Lima K, Loureiro SR, Crippa JA. Mental health in medical residents: relationship with personal, workrelated, and sociodemographic variables. Rev Bras Psiquiatr [Internet]. 2016 [acesso 31 jul 2019];38(4):318-24. DOI: 10.1590/1516-4446-2015-1882

39. Portela NLC, Pedrosa AO, Cunha JDS, Monte LRS, Gomes RNS, Lago EC. Síndrome de burnout em profissionais de enfermagem de urgência e emergência. Rev Pesqui [Internet]. 2015 [acesso 16 set 2020];7(3):2749-60. DOI: 10.9789/2175-5361.2015.v7i3.2749-2760

40. Santos ER, Neri LV, Wanderley ELS. Síndrome de burnout em fisioterapeutas de um hospital público de alta complexidade da cidade do Recife, Pernambuco. Acta Fisiátrica [Internet]. 2018 [acesso 31 jul 2019];25(1):31-5. DOI: 10.11606/issn.2317-0190.v25i1a158832 
41. Silva JLL, Soares RS, Costa FS, Ramos DS, Lima FB, Teixeira LR. Fatores psicossociais e prevalência da síndrome de burnout entre trabalhadores de enfermagem intensivistas. Rev Bras Ter Intensiva [Internet]. 2015 [acesso 14 maio 2019];27(2):125-33. DOI: 10.5935/0103-507X.20150023

42. Silva ATC. Esgotamento profissional e depressão em profissionais da estratégia saúde da família no município de São Paulo [tese] [Internet]. São Paulo: Universidade de São Paulo; 2015 [acesso 14 maio 2019]. DOI: 10.11606/T.5.2015.tde-27102015-084632

43. Silva SCPS, Nunes MAP, Santana VR, Reis FP, Machado Neto J, Lima SO. A síndrome de burnout em profissionais da rede de atenção primária, Brasil. Ciênc Saúde Coletiva [Internet]. 2015 [acesso 14 maio 2019];20(10):3011-20. DOI: 10.1590/1413-812320152010.19912014

44. Silveira ALP, Colleta TCD, Ono HRB, Woitas LR, Soares SH, Andrade VLÂ, Araújo LA. Síndrome de burnout: consequências e implicações de uma realidade cada vez mais prevalente na vida dos profissionais de saúde. Rev Bras Med Trab [Internet]. 2016 [acesso 16 set 2020];14(3):275-84. DOI: 10.5327/ Z1679-443520163215

45. Souza EOR. Qualidade de vida dos profissionais de enfermagem que atuam em unidades pediátricas de hospitais escola do município de Belo Horizonte [dissertação] [Internet]. Belo Horizonte: Universidade Federal de Minas Gerais; 2017 [acesso 16 set 2020]. Disponível: https://bit.ly/2Yy5NRY

46. Tironi MOS, Teles JMM, Barros DS, Vieira DFVB, Silva Filho CM, Martins DF Jr et al. Prevalência de síndrome de burnout em médicos intensivistas de cinco capitais brasileiras. Rev Bras Ter Intensiva [Internet]. 2016 [acesso 14 maio 2019];28(3):270-7. DOI: 10.5935/0103-507X.20160053

47. Vasconcelos EM, Martino MMF, França SPS. Burnout and depressive symptoms in intensive care nurses: relationship analysis. Rev Bras Enferm [Internet]. 2018 [acesso 14 maio 2019];71(1):135-41. DOI: 10.1590/0034-7167-2016-0019

48. Vidotti V, Ribeiro RP, Galdino MJQ, Martins JT. Síndrome de burnout e o trabalho em turnos na equipe de enfermagem. Rev Latinoam Enferm [Internet]. 2018 [acesso 14 maio 2019];26:e3022. DOI: 10.1590/ $1518-8345.2550 .3022$

49. Zampieri FG. Brazilian intensivists: exhausted, but (still) happy with their choice? Rev Bras Ter Intensiva [Internet]. 2016 [acesso 14 maio 2019];28(3):215-6. DOI: 10.5935/0103-507X.20160047

50. Zanatta AB, Lucca SR. Prevalence of burnout syndrome in health professionals of an onco-hematological pediatric hospital. Rev Esc Enferm USP [Internet]. 2015 [acesso 14 maio 2019];49(2):253-8. DOI: 10.1590/ S0080-623420150000200010

51. Zavalis A, Vianna LAM, Velasque LS, Schutz V, Machado DA. The influence of stress factors on the attention levels of nursing professionals. Rev Pesqui [Internet]. 2015 [acesso 31 jul 2019];7(4):3375-87. DOI: 10.9789/2175-5361.2015.v7i4.3375-3387

52. Brevidelli MM, Cianciarullo TI. Níveis de adesão às precauções-padrão entre profissionais médicos e de enfermagem de um hospital universitário. Online Braz J Nurs [Internet]. 2006 [acesso 31 jul 2019];5(1):106-15. DOI: 10.5935/1676-4285.2006291

53. Bianchi ERF. Enfermeiro hospitalar e o stress. Rev Esc Enf USP [Internet]. 2000 [acesso 16 set 2020];34(4):390-4. DOI: 10.1590/S0080-62342000000400011

54. World Health Organization. International statistical classification of diseases and related health problems (ICD): ICD-11 [Internet]. Geneva: WHO; 2018 [acesso 31 jul 2019]. Disponível: https://bit.ly/2MpZCgx

55. Kovaleski DF, Bressan A. Síndrome de burnout em profissionais de saúde. Saúde Transform Soc [Internet]. 2012 [acesso 31 jul 2019];3(2):107-13. Disponível: https://bit.ly/3th6EVn

56. Dejours C. Por um novo conceito de saúde. Rev Bras Saúde Ocup [Internet]. 1986 [acesso 16 set 2020];14(54):7-11. Disponível: https://bit.ly/3tmuDCw

57. Dejours C. A loucura do trabalho: estudo de psicopatologia do trabalho. São Paulo: Oboré; 1987.

58. Tironi MOS, Nascimento Sobrinho CL, Barros DS, Reis EJFB, Marques Filho ES, Almeida A et al. Trabalho e síndrome da estafa profissional (síndrome de burnout) em médicos intensivistas de Salvador. Rev Assoc Méd Bras [Internet]. 2009 [acesso 8 out 2019];55(6):656-62. DOI: 10.1590/S0104-42302009000600009 
59. Seligmann-Silva E. Trabalho e desgaste mental: o direito de ser dono de si mesmo. São Paulo: Cortez; 2011. 60. Donoso MTV. Gênero e suas possíveis repercussões na gerência de enfermagem. Reme [Internet]. 2000 [acesso 31 jul 2019];4(1-2):67-9. Disponível: https://bit.ly/39FMDQL

61. Organização Mundial da Saúde. Classificação estatística internacional de doenças e problemas relacionados à saúde: CID-10. $3^{\text {a }}$ ed. São Paulo: Edusp; 1996.

Layla Thamm Jarruche - Especialista - thamm.layla@gmail.com

(D) 0000-0001-9859-1811

Samantha Mucci - Doutora - sammucci@gmail.com

(D) 0000-0003-3809-8173

Correspondência

Layla Thamm Jarruche - Rua dos Jacintos, 372, apt. 66 CEP 04049-050. São Paulo/SP, Brasil.

Participação dos autores

Layla Thamm Jarruche realizou a revisão bibliográfica e redigiu o artigo. Samantha Mucci orientou e supervisionou o projeto e elaborou as conclusões.

Recebido: 18.10 .2019

Revisado: 8.12 .2020

Aprovado: $\quad 6.1 .2021$ 